\title{
Cooperative Distribution Algorithm of Green Supply Chain Considering the Risk Aversion of Manufacturers
}

\author{
Han Fang $^{1}$ and Yang Yongqiang ${ }^{1}$ \\ 1. College of Mechanical \& Electrical Engineering, ShaanXi University of Science \\ \& Technology, Xi'An, China \\ 'hanfang@sust.edu.cn
}

\begin{abstract}
Considering production and marketing coordination between supply chain enterprises, this paper specifies a multi-agent supply chain concurrent negotiation model for twostage supply chain in the supply chain environment. Coordinator is used to search optimal proposals based on particle swarm optimization (PSO) and send these proposals to other threads. Proposal strategies based on retention value and time are put forward by learning retention value of rivals through Parzen-minaow estimation. Simulation experiment is conducted to test negotiation performance of the model. Compared with ordinary models, the model makes two improvements as follows. (1)PSO is inserted into coordinator to improve negotiation efficiency; (2) Proposal strategies are effectively supported by Parzen-window estimation and enable agents to consider the retention values of rivals. Further researches need to be done to study trust relationship between agents and influence of external environmem on supplyain negotiation solution.
\end{abstract}

Keywords: Particle swarm Coptimization (RSO); Estimation, supply chain; Coordination; Negotiation

\section{Introduction}

Intelligent management of supply chains is an effective way for enterprises to save costs and improve competitrveness in the market environment of complicated competition and dynamic demands. Multi-agent system has advantages such as distributivity, interactivicy and intellectuality, so it is suitable for enterprisespanning management of supply chains in the complicated market environment. In an environment of distributive supply chain, enterprises hope to concurrently negotiate with more than one supplier to get better products, services and profits, meeting their own demands while improving collaborative efficiency of supply chains.

We found that most negotiation models require buyer agents to give counterproposals after receiving proposals from all seller agents. This pattern restricts information exchange and flexibility of negotiation strategies. In addition, most moaels pay little attention to efficiency of coordinators. In the actual operation of supply chains, manufacturers look forward to consensus and win-win with sellers through timely negotiation. For this reason, this paper establishes a multi-agent concurrent model for two-stage supply chains. That means manufacturer agents can use mixed proposal strategies based on retention values and time to negotiate with seller agents through more than one concurrent threads. Meanwhile, PSO is used to coordinate each concurrent negotiation threads to support negotiation agents to reach agreements within limited time. 


\section{Concurrent Negotiation Model}

\subsection{Model Framework}

For accepting or refusing proposals and giving counter-proposals. This paper takes sellers and manufacturers as research objectives and supply chain as a production and marketing coordination network. In this model, manufacturers provides products and sellers need to purchase. A model framework describing that one manufacture agent concurrently negotiate with several seller agents is illustrated in Figure 1. Manufacturers and sellers have their own negotiation information (such as value range of negotiation issues and negotiation deadlines) and they do not know the information of each other. Manufacturer agents are decomposed into several sub-agents and one coordinator. The coordinator establishes negotiation threads at the same number with current seller agents and formulates different negotiation strategies for each thread. Each sub-agent is controlled through corresponding negotiation strategy. Coordinator needs to flexibly deal with negotiation thread information with different time and statues and timely update confidence values of other sub-agents because each seller agent may have different proposalstrategies. Then sub-agents negotiate with seller agents based on updated confidence values. Negotiation threads include sub-agents and corresponding sellen agents, and reserve negotiation information of manufacturer agents. They are also responsible

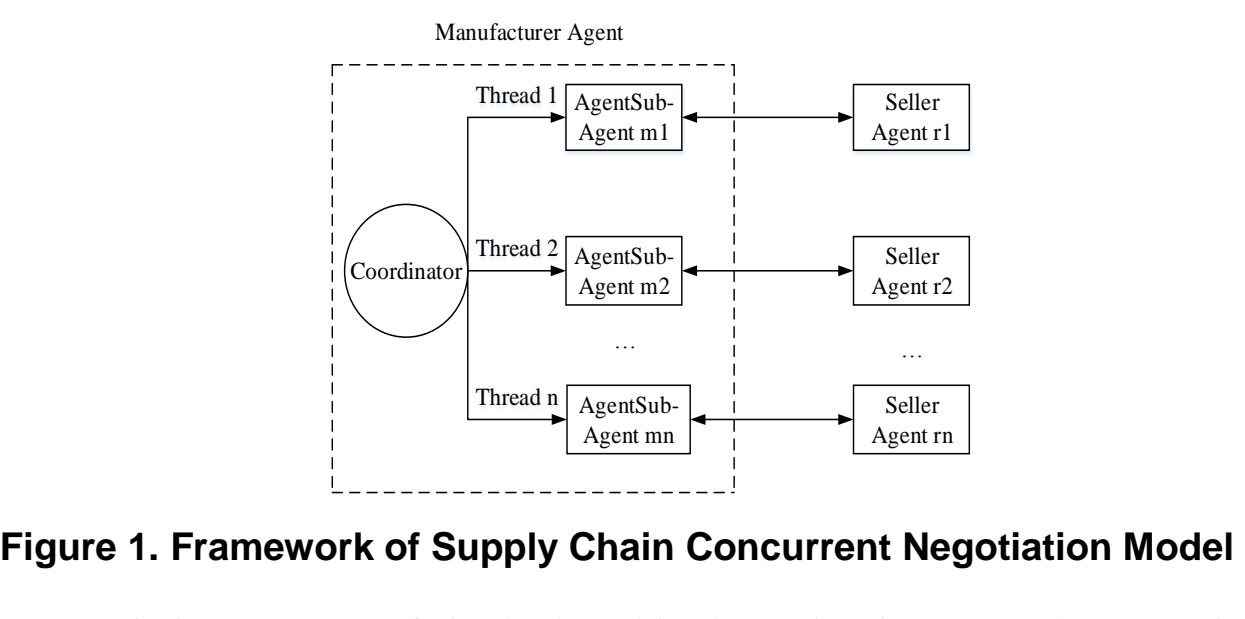

The negotiation progress of single thread is shown in Figure 2. When negotiation starts, coordinator inimalizes negotiation thread according to the number of seller agents. Then inlaunches negotiation request and seller agents give response. All threads are carnied out continuously. Check if there is the best proposal from coordinator before starting next round of proposing. If there is no best proposal, send out counter-proposals according to negotiation strategies. If there is the best proposal, judge whether utility value of the best proposal is bigger than that of curent proposal. If yes, negotiation belief of manufacture agents need to be updated. If no, current negotiation strategy remains the base for giving proposal (counter-proposal). When sub-agents and supplier agent success or fail in negotiating, each thread will send negotiation results back to the coordinator. 


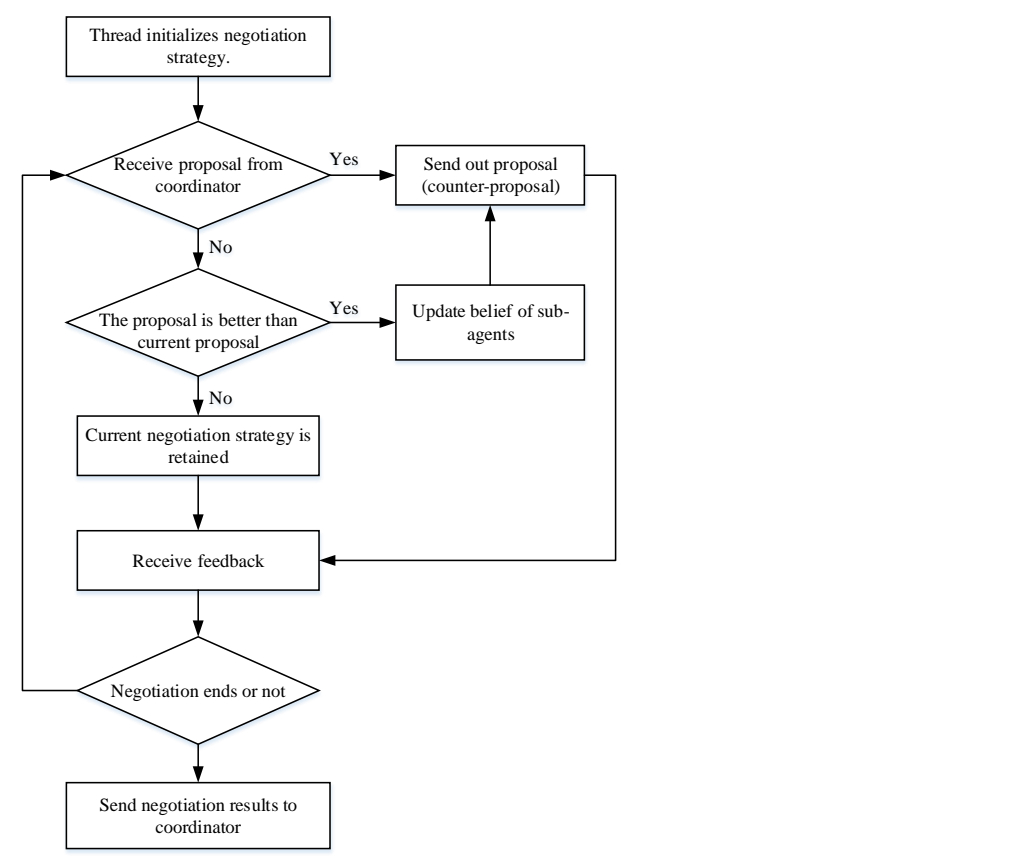

Figure 2. Negotiation Process of Single Thread

\subsection{Setup of Major Parameters}

$A g=\left\{m, r_{1}, \cdots, r_{n}\right\}$ denotes a set of one manufacturer agent and several seller agents. For $a \in A g, T_{\text {man }}^{a}$ represents the deadline of agent $a$. $X \in\left\{V_{t}^{a_{i} \rightarrow a_{j}}, \min _{i}^{a}, \max _{i}^{a}, w_{i}^{a}\right\}$ denotes negotiatign topics, where $V_{t}^{a_{i} \rightarrow a_{j}}$ represents the proposal values the agent $a_{i}$ sends to agent $a_{j}$ at the time of $t$, and $\left[\min _{i}^{a}, \max _{i}^{a}\right]$ represents value range $f$ agent $a$ for topic $i . W_{i}^{a}$ denotes the weight vector of agent $a$ for topic $i$ represents actions of negotiation. In $A \in\{$ accept, reject, propose $\}$ "accept" means accepting proposal; "reject" means refusing proposal and "propose" means giving other proposals. $U$ is utility function. Quantitative topic $v_{i}$ is evaluation function

$$
u^{a}\left(v_{i}\right)=\left\{\begin{array}{l}
\frac{\max ^{a}}{\max ^{a}-\min _{i}^{a}} \\
\text { Preference diminishing for topic } i \\
\frac{v_{i}-\min _{i}^{a}}{\max _{i}^{a}-\min _{i}^{a}} \\
\text { Preference ascending for topic } i
\end{array}\right.
$$

Overall utility function of topics

$$
U^{a}=\sum_{i=1}^{n} w_{i}^{a} u^{a}\left(v_{i}\right)
$$

Final united utility function is the utility sum of manufacturer and seller when a thread negotiate results. It can be expressed as:

$U^{A L L}=\lambda U^{m}+(1-\lambda) U^{r}$ 
If $S$ is used to denote proposal strategy, multi-agent supply chain concurrent negotiation model is a six-tuple $\Gamma=\{A g, X, T, A, U, S\}$.

\subsection{Proposal Strategies}

2.3.1. Time-based Proposal Strategy: Time-based concession strategy was proposed by Faratin who believed that time is one of the decisive factors to concession range.

Time-based proposal strategy of manufacturer for the topic $v_{i}$ at the time of $t$ can be expressed as:

$$
V^{m \rightarrow r}(t)_{f}=\left\{\begin{array}{l}
\min _{i}^{m}+\left(t / T_{\max }^{m}\right)^{B}\left(\max _{i}^{m}-\min _{i}^{m}\right), \\
\text { Preference diminishing for topic } i \\
\max _{i}^{m}-\left(t / T_{\max }^{m}\right)^{B}\left(\max _{i}^{m}-\min _{i}^{m}\right), \\
\text { Preference ascending for topic } i
\end{array}\right.
$$

Time-based proposal strategy of seller for the topic $v_{i}$ at the time of $t$ can be expressed as:

$$
V^{r \rightarrow m}(t)_{f}=\left\{\begin{array}{l}
\min _{i}^{r}+\left(t / T_{\max }^{r}\right)^{B}\left(\max _{i}^{r}-\min _{i}^{r}\right), \\
\text { Preference diminishing for topic } i \\
\max _{i}^{r}-\left(t / T_{\max }^{r}\right)^{B}\left(\max _{i}^{r}-\min _{i}^{r}\right), \\
\text { Preference ascending for topic } i
\end{array}\right.
$$

2.3.2. Proposal Strategy based on Estimation of Rival Retention Value for Topics: Parzen window estimation is usually used to estimate probability density function of unknown distribution. The basic ldea is to estimate the overall density function according to the average density of each point in a certain range. Taking manufacturer agents for example, calculate seller agents' retention values for topics. Assume $\left(x_{1}, x_{2}, \cdots, x_{N}\right)$ is the sample on unknown distribution and $x_{i}$ is the proposed value of seller agent for atopic. The distribution can be estimated as follows:

$$
\hat{f}_{h}(x)=\frac{1}{N \cdot h} \sum_{i=1}^{N} \rho\left(\frac{-x_{i}}{h}\right)
$$

Where Nenotes the sample size, $h$ is window width and $\varphi(\cdot)$ is window function. For the mand function, it is usually uniform function, trigonometric function and gamma function. The model employs Gaussian function. Therefore, the probability density function of seller agent's proposal strategy can be expressed as:

$$
P(x)=\frac{1}{N \cdot(3 \pi)^{d / 2} \cdot h} \sum_{i=1}^{N} \exp \left\{-\frac{\left(x-p_{r i}\right)^{2}}{2 h^{2}}\right\}
$$

Where $d$ represents characteristic space dimension and $p_{r i}$ denotes the proposed values of seller agent in the negotiation round $i$.

Based on this probability density function, manufacturer agent may give counterproposal to seller while retaining estimation values on the premise of safeguarding own benefits.

$$
V^{m \rightarrow r}(t)_{s}=\left\{\begin{array}{l}
\min _{i}^{m}, \quad \min _{i}^{m}<\int P(x) x d x \\
e^{t / T} \times \int P(x) x d x, \quad \text { otherwise }
\end{array}\right.
$$


2.3.3. Lineary Weighted Proposal Strategy: Above two proposals are complementary to each other to some extent. Time-based proposal strategy fails to take into account the rival retention values for topics though it considers the satisfaction degree of negotiation deadline; while the proposal strategy based on estimation of rival retention value for topics ignores the constraint of negotiation deadline. Thus these two proposal strategies are combined by dynamic linear weight:

$$
\begin{aligned}
V^{m \rightarrow r}(t) & =s_{1} \times V^{m \rightarrow r}(t)_{s}+s_{2} \times V^{m \rightarrow r}(t)_{f} \\
s_{1}, s_{2} & \in[0,1], \text { 且 } s_{1}+s_{2}=1 。
\end{aligned}
$$

Where $s_{1} 、 s_{2}$ denote integration coefficient under the condition of $s_{1}, s_{2} \in[0,1]$ and $s_{1}+s_{2}=1$.

\section{PSO-based Coordination Strategy}

In order to manage concurrent negotiation threads, PSO algorithm is inse ted into the coordinator. Search the best proposal and send it to other threads still under negotiation until all threads are finished or reach the negotiation deadline. During concurrent negotiation, one thread is over when it reaches greement

PSO is a population search algorithm based on smulation of preation of bird flock. Unlike genetic algorithm, it has no crossover and mutation operators. It searches according to rate. In this algorithm, an Individual searches the optimal solution for itself or for the near individuals. This population behavior also searches the globally optimal solution for the whole space. Assume $x_{k}^{t}$ represents location of the individual $k$ at the time of $t$ and $k$ moves at the rate of $u_{k}^{t+1}$. It can be expressed as:

$x_{k}^{t+1}=x_{k}^{t}+u_{k}^{t+1}$

Rate is an important parameterand expressed as:

$$
u_{k_{j}}^{t+1}=u_{k_{j}}^{t}+c_{1} \cdot r_{1 j}^{t} \cdot\left(y_{k j}^{t}-x_{k j} c_{2} \cdot r_{2 j}^{t} \cdot\left(y_{k j}-x_{k j}^{t}\right)\right.
$$

Where $u_{k_{j}}^{t}$ denotes the rate of jind vidual $k$ at the time of $t$ in dimension $j$; denotes location of individual $k$ at the time of $t$ in dimension $j ; c_{1}$ and $c_{2}$ are used to accelerate and larger than $0 ; r_{1 j}^{t}$ and $r_{2 j}^{t}$ represent random numbers in the internal $[0,1] ; y_{k j}^{t}$ is the opfintal solution for individual and $\hat{y}_{k j}^{t}$ is the globally optimal solution. The optimal solution for the individual $t+1 \mathrm{can}$ be expressed in formula (5).

$y_{k}^{t+1}=\left\{\begin{array}{cc}y_{k}^{t} & \text { if } f\left(x_{k}^{t+1}\right) \leq f\left(y_{k}^{t}\right) \\ t+1 & \text { if } f\left(x_{k}^{t+1}\right)>f\left(y_{k}^{t}\right)\end{array}\right.$

Where $f(\cdot)$ is fitness function, thus the optimal solution for the whole space is:
$f\left(\hat{y}_{k}^{t}\right)=\max \left\{f\left(y_{1}^{t}\right), f\left(y_{2}^{t}\right), \cdots f\left(y_{N}^{t}\right)\right\}$

According to features of PSO algorithm and supply chain concurrent negotiation model, taking manufacturer agent as an example, negotiation threads can be taken as particles in PSO algorithm. Proposals between threads represent current location of particles, and proposal successfully negotiated through a thread is the optimal solution for particle. The optimal solution with highest utility value is the globally optimal solution among all successfully negotiated threads. Increment or decrement of proposal values is rate and the fitness function is utility function $U^{m}$. 


\section{Description of Negotiation Procedures}

Based on methods above, negotiation procedures can be described as below:

Step 1: Coordinator establishes negotiation threads according to the number of sellers and initializes proposal strategies and PSO parameters of each thread. PSO is used to coordinate concurrent negotiation threads. Calculate the best proposal and send it to other sub-agents.

Step 2: When initializing, manufacturer agents and seller agents use time-based proposal strategy to negotiate with sellers according to the formula (4) and (5).

Step 3: When the two parties accumulate some history data of negotiation after a certain period, the formula (6) and (7) are used to calculate retention values. Linearly weighted proposal strategy can be used to conduct one-to-one negotiation according to the formula (9).

Step 4: After sub-agents receive the best proposal from coordinator, current proposal strategy can be retained if the utility value of the proposal is larger than that of their own proposal. Otherwise, is appointed as new rate to calculate the concession parameter of time-based proposal strategy $\beta^{\prime}=\frac{\ln \left(\max _{i}^{m}-\min _{j}^{m}\right)-\ln u^{\prime}}{\ln (t+1)-\ln t}$ through the formula (3) and (9). If negotiation is successful, move forward to Step 5; otherwise, return to Step 3.

Step 5: When one thread's negotiation is successful, send negotiation results to coordinator and wait until other threads complete negotiation.

Step 6: Coordinator chooses the optimal seller accôrding to the formula (3) when all threads complete negotiation.

\section{Experiment}

In order to verify the rationality andescientificity of the model, simulation experiment is conducted by setting corresponding parameters. For comparative purpose, experiment parameters are same with the reference [4] as shown in Table 1. The experiment should be repeated for 3 times under same condition with average values as experimental results. The experimental evaluation criteria include final united utility fynction, sucoess rate of negotiation and average negotiation time (average ratio of actual time of successful negotiation to negotiation deadline)

Tabje 1. Experiment Parameter Setting

\begin{tabular}{ccc} 
& Description & Value \\
\hline & Number of seller agents & {$[1,30]$} \\
& Number of topics & {$[1,8]$} \\
$\min _{i}^{a}$ & Minimum value of agent $a$ to topic $i$ & {$[0,20]$} \\
$\max _{i}^{a}$ & Maximum value of agent $a$ to topic $i$ & {$[30,50]$} \\
$w_{i}^{a}$ & Weight of agent $a$ to topic $i$ & $1 / \mathrm{N}$ \\
\hline
\end{tabular}

According to results of simulation experiment (as shown in Figure 3,4,5), the model achieves a total utility value of 0.452 , an average negotiation time of 0.72 and a success rate of $78.5 \%$ when the number of sellers is 5 . Ordinary models have total utility value of 0.395 , average negotiation time of 0.79 and success rate of $70.9 \%$. When the number of sellers is 10 , the model achieves a total utility value of 0.516 , an average negotiation time of 0.76 and a success rate of $84.6 \%$ while ordinary models have total utility value of 0.427 , average negotiation time of 0.83 
and success rate of $73.5 \%$. The model also achieves better negotiation results than ordinary models when the number of sellers is 15, 20, 25 and 30. In addition, the proposal strategy used in this model provides the best proposal for other threads. As the negotiation goes on, the rest threads refuse to accept proposals that are beyond their own preference ranges. Therefore, average negotiation time reduces and growth of success rate decelerates with increasing number of sellers.

Analysis above gets following conclusions: (1) the model can achieve negotiation results with larger utility values compared with ordinary models, and utility value increases with rising number of sellers. This indicates that negotiation result will be better when negotiating with more sellers if costs are not considered. (2) The model takes less time to reach negotiation results than ordinary models. When negotiating with same number of sellers, the model achieves results more quickly. PSO algorithm is used to accelerate concession rate, shorten negotiation time and improve negotiation efficiency. (3) The model has a higher success rate of negotiation than ordinary models. Parzen window estimation is used to learn retention values of rivals for topics, to gain more negotiation information, o help own parties to choose more suitable negotiation strategies and to improve success rate.

Figure 3. Final Total Utility Values for Different Number of Sellers

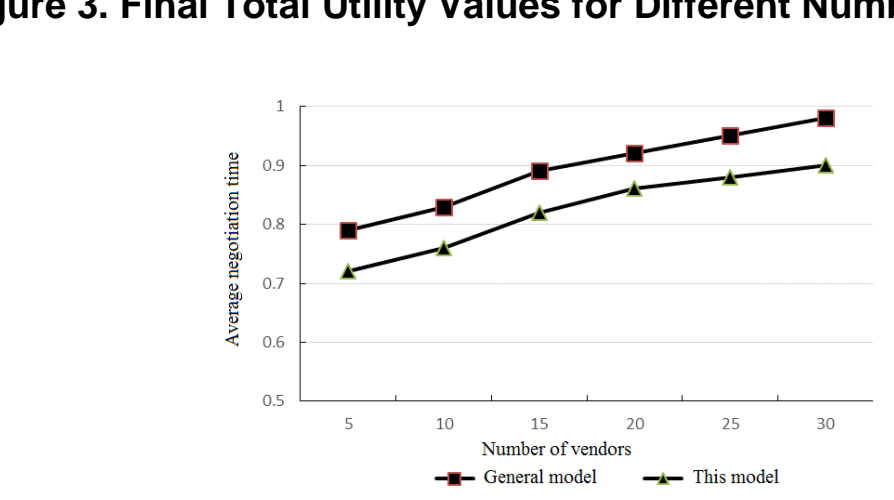

Figure 4. Average Negotiation Time for Different Number of Sellers 


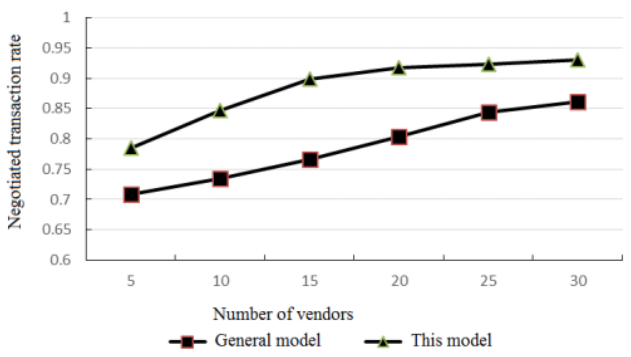

Figure 5. Negotiation Success Rate for Different Number of Sellers

\section{Conclusion}

Considering production and marketing coordination between supply chain enterprises, this paper specifies a concurrent negotiation model under constrant of poor information and applies multi-agent technology to two-stage upply chain. PSO-based negotiation strategy can update belief values of/agents during negotiation so that negotiation can be carried out continuously. Simulation experiment results indicates that the model is feasible and efficient and has advantages in utility values of negotiation results, negotiation time and success rate compared with other concurrent negotiation models.

\section{References}

[1] T. Su, W. Wang, Z. Lv, "Rapid Delaunay criangulation for randomly distributed point cloud data using adaptive Hilbert curve[J]", Computers \& Graphics, (2016), 54. pp. 65-74.

[2] J. Hu, Z. Gao and W. Pan. "Multiangle Social Network Recommendation Algorithms and Similarity Network Evaluation[J]", Journal of Applied Mathematics, 2013 (2013).

[3] S. Zhou, L.Mi, H. Chen, Y. Geng, Building dêtection in Digital surface model, 2013 IEEE International Conference on Imaging Systems and Techniques (IST), Oct.(2012).

[4] J. He, Y.Geng, K. Pahlayan, "Toward Accurate Human Tracking: Modeling Time-of-Arrival for Wireless Wearable Sensors in Multipath Environment", IEEE Sensor Journal, vol.14, no. (11), pp. 3996-4006, Nov (2014).

[5] Z. Lv, A. Halawant, S. Fen, "Touch-less Interactive Augmented Reality Game on Vision Based Wearable Devee 1 ", Personal and Ubiquitous Computing, (2015), vol. 19, no. 3, pp. 551-567.

[6] G. Bao, L. Mi, Y. Geng M. Zhou, K. Pahlavan, "A video-based speed estimation technique for localizing the wireless capsule endoscope inside gastrointestinal tract", 2014 36th Annual International Conference of the IEEE Engineering in Medicine and Biology Society (EMBC), Aug. (2014).

[7] D. Zeng, Y. Geng, Content distribution mechanism in mobile P2P network", Journal of Networks, vol. 9, no. 5, pp. 1229 1236, Jan. (2014).

[8] G. W, Lv Z, Hao M. "Change detection method for remote sensing images based on an improved Markov random field[J]", Multimedia Tools and Applications, (2015), pp. 1-16.

[9] Z. Chen, W. Huang, Z. Lv, "Towards a face recognition method based on uncorrelated discriminant sparse preserving projection[J]", Multimedia Tools and Applications, (2015), pp.1-15.

[10] J. Hu and Z. Gao, "Distinction immune genes of hepatitis-induced heptatocellular carcinoma[J]",Bioinformatics,(2012), vol. 28, no. 24, pp. 3191-3194.

11. T. Su, W. Wang, Z. Lv. "Rapid Delaunay triangulation for randomly distributed point cloud data using adaptive Hilbert curve[J]”, Computers \& Graphics, (2016), 54: pp. 65-74.

[12] W. Gu, Z. Lv, M. Hao, "Change detection method for remote sensing images based on an improved Markov random field[J]", Multimedia Tools and Applications, (2015), pp. 1-16.

[13] Z. Lv, A. Tek, Da Silva F. Game on, science-how video game technology may help biologists tackle visualization challenges[J]. PloS one, (2013), vol. 8, no. 3, 57990.

[14] Z. Chen, W. Huang, Z. Lv, "Towards a face recognition method based on uncorrelated discriminant sparse preserving projection[J]", Multimedia Tools and Applications, (2015),pp.1-15.

[15] D. Jiang, X. Ying, Y. Han, "Collaborative multi-hop routing in cognitive wireless networks[J]", Wireless Personal Communications, (2015), pp. 1-23.

[16] Z. Lv, A. Tek, Da Silva F. Game on, science-how video game technology may help biologists tackle visualization challenges[J]. PloS one, (2013), vol. 8, no. 3, 57990. 
[17] J. D, Z. Xu, Z. Lv, "A multicast delivery approach with minimum energy consumption for wireless multi-hop networks[J]", Telecommunication Systems, (2015), pp. 1-12.

[18] C. Fu, P. Zhang, J. Jiang, "A Bayesian approach for sleep and wake classification based on dynamic time warping method[J]", Multimedia Tools and Applications, (2015), pp.1-20.

[19] Z. Lv, "Wearable smartphone: Wearable hybrid framework for hand and foot gesture interaction on smartphone[C]", //Computer Vision Workshops (ICCVW), 2013 IEEE International Conference on. IEEE, (2013): pp. 436-443.

[20] Y. Lin, J. Yang, Z. Lv, "A Self-Assessment Stereo Capture Model Applicable to the Internet of Things[J]”, Sensors, (2015), vol. 15, no. 8, pp. 20925-20944.

[21] J. Yang, S. He, Y. Lin, "Multimedia cloud transmission and storage system based on internet of things[J]", Multimedia Tools and Applications, (2015), pp. 1-16.

[22] Z. Lv, T. Yin, Y. Han. WebVR—web virtual reality engine based on P2P network[J]. Journal of Networks, (2011), vol. 6, no. 7, pp. 990-998.

[23] J. Yang, S. He, Y. Lin, "Multimedia cloud transmission and storage system based on internet of things[J]", Multimedia Tools and Applications, (2015).

[24] C. Guo, X. Liu, M. Jin, "The research on optimization of auto supply chain network robust model under macroeconomic fluctuations[J]", Chaos, Solitons \& Fractals, (2015).

[25] L. X, Z. Lv, J. Hu XEarth: A 3D GIS Platform for managing massive chy information[C]//Computational Intelligence and Virtual Environments for Measurement Systems and Applications (CIVEMSA), 2015 IEEE International Conference on. IEEE, (2015). pp. 1-6.

[26] J. Yang, B. Chen, J. Zhou, "A Low-Power and Portable Biomedical Device for Respiratory Monitoring with a Stable Power Source[J]”, Sensors, (2015), vol. 15, no. 8, pp 19618-19632.

[27] G. Bao, L. Mi, Y. Geng, K. Pahlavan, "A computer vision based speed estimation echnique for localiz ing the wireless capsule endoscope inside small intestine", 36th Amual International Conference of the IEEE Engineering in Medicine and Biology Society (EMBC), Aug. (2014)

[28] X. Song, Y. Geng, "Distributed community detection optimzation algorithm for complex networks", Journal of Networks, vol. 9, no. 10, pp. 2758-2765, Jan. (2014).

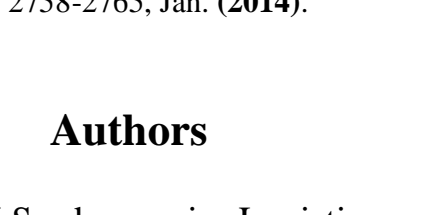

Han Fan, She received her M.S. degree in Logistics engineering from Shaanxi University of Science and Technology in Xi@in, China. She is currently a lecturer in the College of mechanical and electrical engineering at Shaanxi University of Science and Technology. Her research phterest is Manly in the area of Logistics facilities planning, industrial engineering. She has published several research papers in scholarly journals in the above research areas and has participated in several books. 
International Journal of Smart Home

Vol. 10, No. 9 (2016

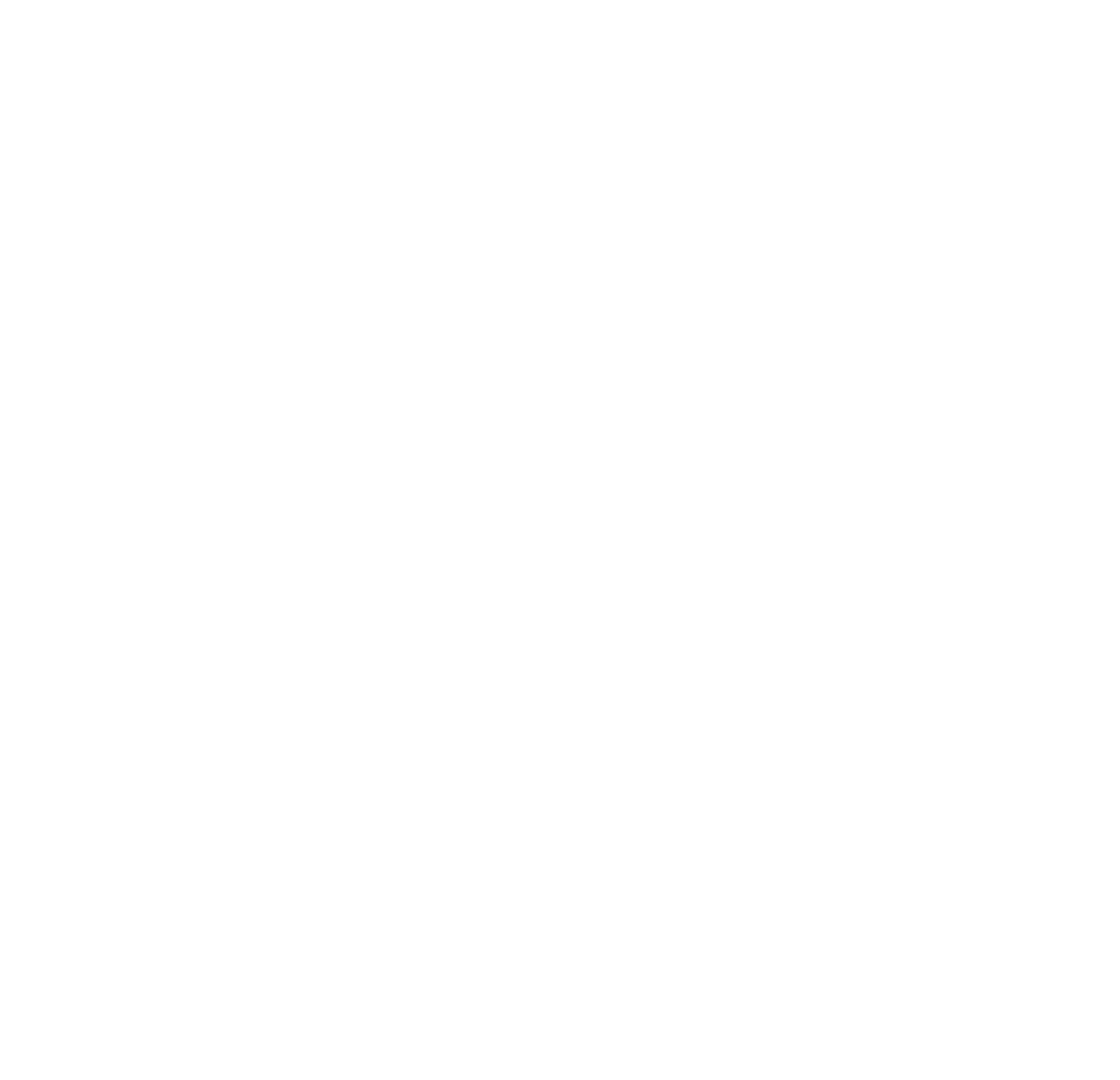

